\title{
Follicular lymphoma presenting with massive splenomegaly
}

\author{
Yosei Fujioka $\cdot$ Mineo Kurokawa
}

Received: 6 September 2011/Revised: 22 November 2011/ Accepted: 22 November 2011/Published online: 14 December 2011

(c) The Japanese Society of Hematology 2011

A 56-year-old male presented with abdominal distension and weight loss. Physical examination detected a giant spleen extending to the pelvic brim with a hard texture on palpation. Laboratory findings included a serum lactate dehydrogenase level of $319 \mathrm{U} / \mathrm{L}$ and a soluble interleukin2 receptor level of $4013 \mathrm{U} / \mathrm{mL}$. Computed tomography scan was remarkable for massive splenomegaly and lymphadenopathy (Fig. 1, left upper panel). F-18 fluorodeoxyglucose positron emission tomography (FDG-PET) revealed increased uptakes in the markedly enlarged spleen and lymph nodes (Fig. 1, middle panel).

Biopsy of the enlarged cervical node led to a diagnosis of follicular lymphoma (Fig. 1, left lower panel). Flow cytometry showed that the neoplastic cells were positive for CD10, CD19, CD20, and CD38. Monotypic expression of kappa light chain was noted. Cytogenetic study disclosed chromosomal abnormalities including $\mathrm{t}(14 ; 18)(\mathrm{q} 32 ; \mathrm{q} 21.3)$, $\mathrm{t}(3 ; 22)(\mathrm{q} 27 ; \mathrm{q} 11.2)$ and $\operatorname{del}(6)(\mathrm{q} 21 \mathrm{q} 23)$ in 19 of 20 metaphases was analyzed. BCL2-IGH fusion signal was detected by fluorescence in situ hybridization analysis. Bone marrow biopsy showed paratrabecular proliferation of lymphoma cells. Reverse transcriptase-polymerase chain reaction for $B C R-A B L 1$ mRNA and sequencing analysis for $J A K 2$ V617F mutation were negative. Given these data, we concluded that the splenomegaly was attributable to infiltration of lymphoma. Treatment with six cycles of rituximab,

Y. Fujioka · M. Kurokawa $(\bowtie)$

Department of Hematology and Oncology,

Graduate School of Medicine, The University of Tokyo,

7-3-1 Hongo, Bunkyo-ku, Tokyo 113-8655, Japan

e-mail: kurokawa-tky@umin.ac.jp

M. Kurokawa

Department of Cell Therapy and Transplantation Medicine,

The University of Tokyo Hospital, Tokyo, Japan cyclophosphamide, doxorubicin, vincristine and prednisolone (R-CHOP) regimen was initiated. F-18 FDG-PET after the chemotherapy showed normalization of the spleen (Fig. 1 , right panel)

To our knowledge, this is the first case of follicular lymphoma manifesting with massive splenomegaly. Among hematological malignancies, massive splenomegaly is usually associated with chronic myeloid leukemia, primary myelofibrosis, chronic lymphocytic leukemia, or splenic marginal zone lymphoma. The present case suggests that follicular lymphoma should also be considered as a cause of massive splenomegaly. 
Fig. 1 Follicular lymphoma with massive splenomegaly. Computed tomography scan at diagnosis (left upper).

Histopathology of the biopsied cervical node (left lower). FDGPET at diagnosis (middle). FDG-PET after chemotherapy (right)
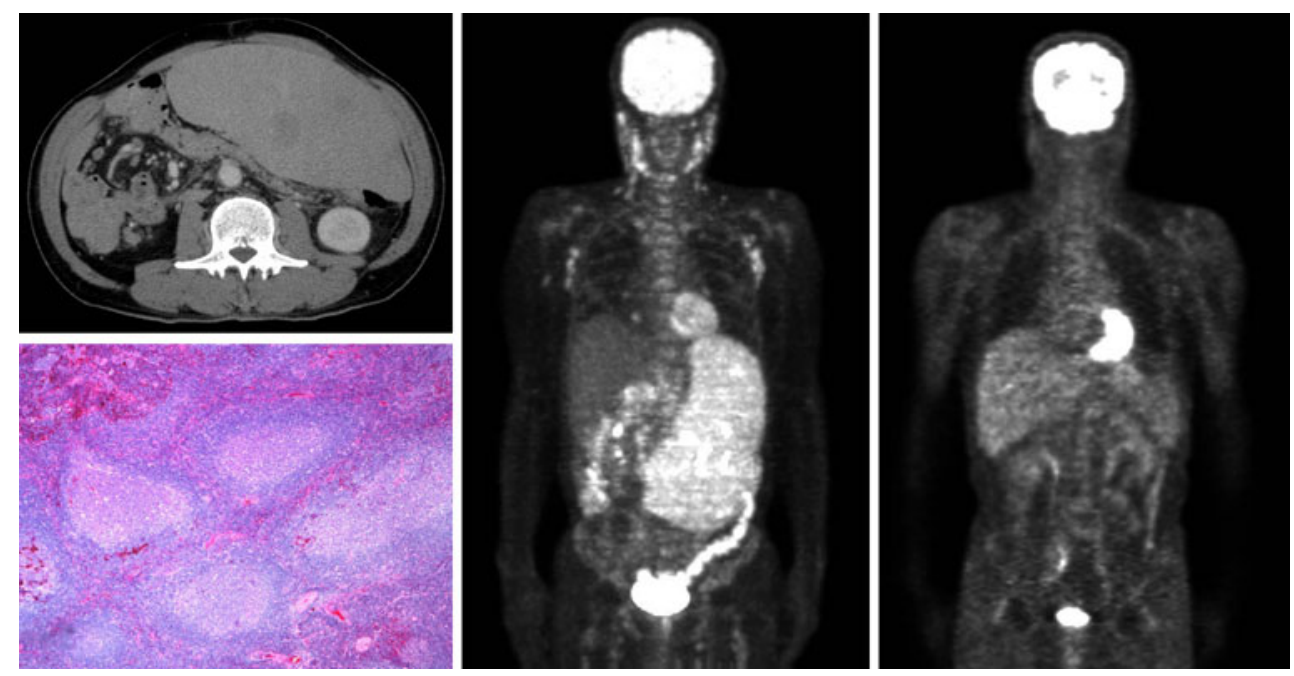

Conflict of interest The authors have no conflicts of interest. 